\title{
Performance Improvement in Binarization for Fingerprint Recognition
}

\author{
Ms. Meghna B. Patel ${ }^{1}$, Dr. Satyen M. Parikh ${ }^{2}$, Dr. Ashok R. Patel ${ }^{3}$ \\ ${ }^{I}$ Assistant Professor, AMPICS, Ganpat University, Kherva, India \\ ${ }^{2}$ Dean, FCA, Ganpat University, Kherva, India \\ ${ }^{3}$ Director, FCA, Hemchandracharya North Gujarat University, Patan, India
}

\begin{abstract}
Fingerprint recognition have gain great attentiveness in the world of biometric. The performance of this system is become worst if the image quality is not good. Pre-processing steps enhance the quality of an image and give the suitable outcome. Image binarization is one of the fundamental pre-processing steps in fingerprint recognition. The purpose of image binarization is dividing an image into foreground and background using thresholding technique. This paper describes the use of global and local thresholding techniques. As well as present the comparative analysis of global, local thresholding and proposed adaptive local thresholding algorithm. The implementation is done using java with FVC2000 and FingerDOS databases of fingerprint and evaluates the best performance of the binarization algorithm using comparing the different criteria like execution time, quality of an image, and window size of neighborhood.
\end{abstract}

\section{Introduction}

Fingerprint Recognition is powerful biometric system available for verification and identification of trusted authentication. It is the most famous biometric technique because of uniqueness, acceptability and persistent characteristics of finger [1,2]. A fingerprint is made up of ridges and valleys pattern. This pattern is remaining unique for a specific individual. Now a day, every forensics and law enforcement agency worldwide routinely uses automatic fingerprint recognition systems [1]. Fingerprint recognition follow some pre-processing steps like normalization, orientation estimation, image enhancement, binarization, thinning and post-processing steps like feature extraction, false point removal, core point detection and minutiae matching.

This paper focus on the ridge extraction (binarization) process. Ridge Extraction is the process which is applied after image enhancement process of fingerprint recognition. It is also known as binarization because it covert the 1-bit binary image with 0 and 1 value from 8-bit gray scale image. 0 indicate black color and that is denoted as ridges while 1 indicate as white color denoted as valleys (furrows). Binarization of an image is done by the important technique that is called as thresholding. The aim of this technique is to compare the gray level of each pixel with threshold value to partitioning an image into foreground and background. If the pixel intensity is larger than threshold value then it is consider as 1 (white pixel) otherwise it is consider as 0 (black pixel).

\section{Related Work On Binarization}

There are mainly two ways of Thresholding algorithm [3][4]. One is Simple Thresholding / Static Thresholding/Global Thresholding and another is Local Thresholding/Adaptive Thresholding/Dynamic Thresholding. We have gone through both ways.

The global threshold applicable when the intensity distribution of objects and background pixels are sufficiently distinct. In the global threshold, a single threshold value is used in the whole image. The global threshold has been a popular technique in many years [5-7]. When the pixel values of the components and that of background are fairly consistent in their respective values over the entire image, global thresholding could be used.

During literature survey we found one of the famous global thresholding algorithms is Otsu algorithm [3]. The weakness of an iterative thresholding is defeat by Otsu algorithm using computing the mean value after each step. This method identify the optimal threshold by making use of histogram of the image [8]. Otsu's method is aimed in finding the optimal value for the global threshold. Global thresholding method is not suitable whenever the background enlightenment is not clear.

There is a difficulty that binarization cannot be done by using single intensity threshold because all fingerprint images do not have constant image contrast in the process of making binary images, and even the contrast ratio of the same person's fingerprints varies every time the device is pressed on. Therefore, the dynamic thresholding method is applied depending on image distribution pixel values and through it, the whole image is binarized into the ridge part and non-ridge part. 
Adaptive thresholding typically takes a grayscale or color image as input and, in the simplest implementation, outputs a binary image representing the segmentation. For each pixel in the image, a threshold has to be calculated. If the pixel value is below the threshold it is set to the background value, otherwise it assumes the foreground value. In adaptive thresholding, different threshold values for different local areas are used There are two main approaches to finding the threshold: (i) the Chow and Kaneko [9] approach and (ii) local thresholding. The assumption behind both methods is that smaller image regions are more likely to have approximately uniform illumination, thus being more suitable for thresholding. Adaptive thresholding is used to separate desirable foreground image objects from the background based on the difference in pixel intensities of each region. The drawback of this method is that it is computational expensive and, therefore, is not appropriate for real-time applications. An alternative approach to finding the local threshold is to statistically examine the intensity values of the local neighborhood of each pixel. The statistic which is most appropriate depends largely on the input image. Simple and fast functions include the mean of the local intensity distribution, local adaptive thresholding, on the other hand, selects an individual threshold for each pixel based on the range of intensity values in its local neighborhood. This allows for thresholding of an image whose global intensity histogram doesn't contain distinctive peaks. Chow and Kaneko [9][10] suggest the use of a 7 X 7 window for local thresholding. In their method, the original image is divided into $7 \times 7$ sub images and a threshold is computed for each sub image. However, a threshold is not computed for sub images with unimodal gray level histogram. For X-ray angiograms Fernando and Monro [11] suggest a local thresholding method [14].

\section{Implementation Of Thresholding Algorithms}

We have applied both the global and adaptive thresholding for image binarization.

First, we have gone through global thresholding. It is done using a single threshold value $\mathrm{T}$ for entire image pixels using following equation (1) which is given by (GONZALEZ; WOODS, 2002). Global Thresholding = Choose threshold $\mathrm{T}$ that separates object from background. If $\mathrm{g}(\mathrm{x}, \mathrm{y})$ is a threshold version of $\mathrm{f}(\mathrm{x}, \mathrm{y})$ at some global threshold $\mathrm{T}$.

$$
g(x, y)= \begin{cases}1, & \text { if } f(x, y)>T \\ 0, & \text { if } f(x, y) \leq T\end{cases}
$$

Using this equation we have took some random value of threshold $\mathrm{T}$ and does some trial and error for satisfactory result. See the result images in Appendix-I in which randomly we tried different threshold value. After studying those output we had a sense that if we will try infinite number of trial and error, we will not able to find a standard threshold using simple thresholding so we gave up this way.

Then, implemented famous global thresholding algorithm Otsu algorithm [3]. In this pixel intensities are stored in an array. Threshold value is count by total mean and variance. Either 0 or 1 value is fixed for every pixel based on this threshold. Hence, only one time the image takes change.

The below equations (2) and (3) are count the value of total mean and variance.

Here, two classes $\mathrm{C} 1$ which contains the gray level $[1, \ldots \mathrm{t}]$ and $\mathrm{C} 2$ witch contains the gray levels $[\mathrm{t}+1, \ldots \mathrm{L}]$ are used for bifurcating the pixels.

Therefore, the probability distribution for class $\mathrm{C} 1$ and $\mathrm{C} 2$ are:

$$
\begin{aligned}
& C_{l}: p_{l} / w_{l}(t), \ldots, p_{t} / w_{l}(t) \text { and } \\
& C_{2}: p_{t+1} / w_{2}(t), \ldots, p_{L} / w_{2}(t) \\
& \text { Where } w_{l}(t)=\sum_{i=1}^{1} p_{i} \\
& \text { and } w_{2}(t)=\sum_{i=+1}^{L} p_{i}
\end{aligned}
$$$$
C_{2} ; p_{t+1} / w_{2}(t), \ldots, p_{L} / w_{2}(t)
$$

And for two classes the mean values are 


$$
\mu_{l}=\sum_{\substack{i=l \\ i p_{i}}} / w_{l}(t)
$$

and

$$
\mu_{2}=\sum i p_{i=r+1} / w_{2}(t)
$$

Otsu defined the between-class variance of threshold image using Discriminate Analysis

$$
\sigma_{B}^{2}=w_{1}\left(\mu_{1}-\mu_{\tau}\right)^{2}+w_{2}\left(\mu_{2}-\mu_{\tau}\right)^{2} \text {. }
$$
(7) Otsu tested that the optimum threshold $t *$ is selected so that the between-class variance B is maximized, for bi-level thresholding

$$
t^{*}=\operatorname{Arg} \operatorname{Max}\left\{\sigma_{B}^{2}(t)\right\}
$$

See the output of this algorithm in Appendix-I. It is good but not acceptable as the ridges are removed from some areas, which leads next process in wrong direction. So we have decided to go through adaptive thresholding technique.

Adaptive thresholding is way of dynamic thresh holding technique in which the different thresh hold value for different local areas are used. It is decided according to the pixel wise. Here $\mathrm{T}$ depends on neighborhood of $(x, y)$.

We have decided to use threshold value as a mean value of all the neighbor pixels. Also tried different matrix value like $5 \times 5$ onwards to $15 \times 15$ and then decided to take $9 * 9$ matrix of pixels [Neighbor pixels are the 9x9 matrix means collection of 81 neighbor pixels]. See the below Figure 1. After calculating threshold value, we check the condition that if the pixel value $>$ threshold value then flag it 1 else 0 . This process repeated for all the pixel of the image.

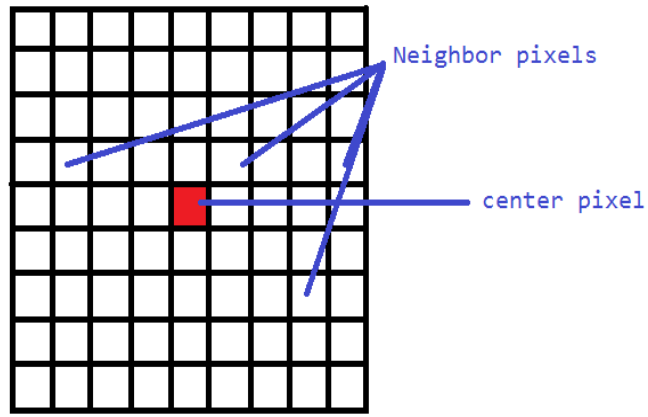

[Figure 1. 9*9 matrix]

The output of adaptive thresholding is quite accepted as the ridge extraction is done nicely. See the result in below in Appendix-I.

\section{Comparative Analysis Of Implemented Algorithms}

The comparative analysis of binarization process is done by the following three evaluation criteria based on the theories of digital printing and the theory of binarization algorithm.

The following criteria were considered in the results:

Criteria-I: Quality of an Image

Criteria-II: Size of the neighborhood in adaptive thresholding

Criteria-III: Execution Time

The implementation of thresholding algorithm is done using java language and for the experiments the databases FVC 2000 DB1, DB2, DB3, DB4 released on the web [12], and FingerDOS database [13] are used. The details of the databases are given in below tables.

\begin{tabular}{|l|l|l|l|l|}
\hline FVC2000 & Sensor Type & Image Size & No. of Impression & Resolution \\
\hline Set B & Low-cost Optical Sensor & $300 \times 300$ & $10 \times 8$ & $500 \mathrm{dpi}$ \\
\hline DB1 & Low-cost Capacitive Sensor & $256 \times 364$ & $10 \times 8$ & $500 \mathrm{dpi}$ \\
\hline DB2 & Optical Sensor & $448 \times 478$ & $10 \times 8$ & $500 \mathrm{dpi}$ \\
\hline DB3 & Synthetic Generator & $240 \times 320$ & $10 \times 8$ & about 500 dpi \\
\hline DB4
\end{tabular}

[Table 1. FVC2000 database][12] 


\begin{tabular}{|c|c|c|c|}
\hline \multicolumn{4}{|l|}{ FingerDOS } \\
\hline Sensor Type & Image Size & No. of Impression & Resolution \\
\hline $\begin{array}{l}\text { optical sensor } \\
\text { (SecuGen iD-USB SC) }\end{array}$ & $260 \times 300$ & $\begin{array}{l}3600=60 \times 6 \times 10 \\
\text { i.e. } \\
\text { No. of subjects }=60 \\
\begin{array}{l}\text { No. of fingers }=6 \\
\text { (index, middle and thumb of right and left } \\
\text { hand) }\end{array} \\
\text { No. of impression }=10\end{array}$ & 500 PPI \\
\hline
\end{tabular}

[Table 2. FingerDOS database][13]

\section{Experimental Results And Analysis}

The below section show the result of the thresholding algorithm based on three different criteria.

\section{Criteria-I: Quality of an Image}

For showing the result we have select 10 fingerprints which are taken from the each cluster of the databases. To measure the quality of an images are based on score ranging from (1) to (5) was used, where (1) is for the worst and (5) is for the best. 15 persons are doing visual analysis of an images based on many characteristics like clarity of ridges, delta, bifurcations, terminations, intersection, spurs and terminations. The average score is considering as final score which is given by 15 persons. The result is shown in Figure 1.

\section{Criteria-II: Size of the neighborhood in adaptive thresholding}

In adaptive thresholding different window size like 5x5, 9x9 and $15 \times 15$ neighborhoods were used to calculate the threshold (T) for each pixel. After doing the visual analysis it proves that $9 \mathrm{x} 9$ window sixe give the best qualitative result. Because when we decrease the size of window the gray scale portion is removed means the important ridge lines are removed and when we increase the size of window it darken the image means extra noise can be added which create false end point and bifurcation minutiae and lead to failure for extracting the true minutiae during feature extraction process.

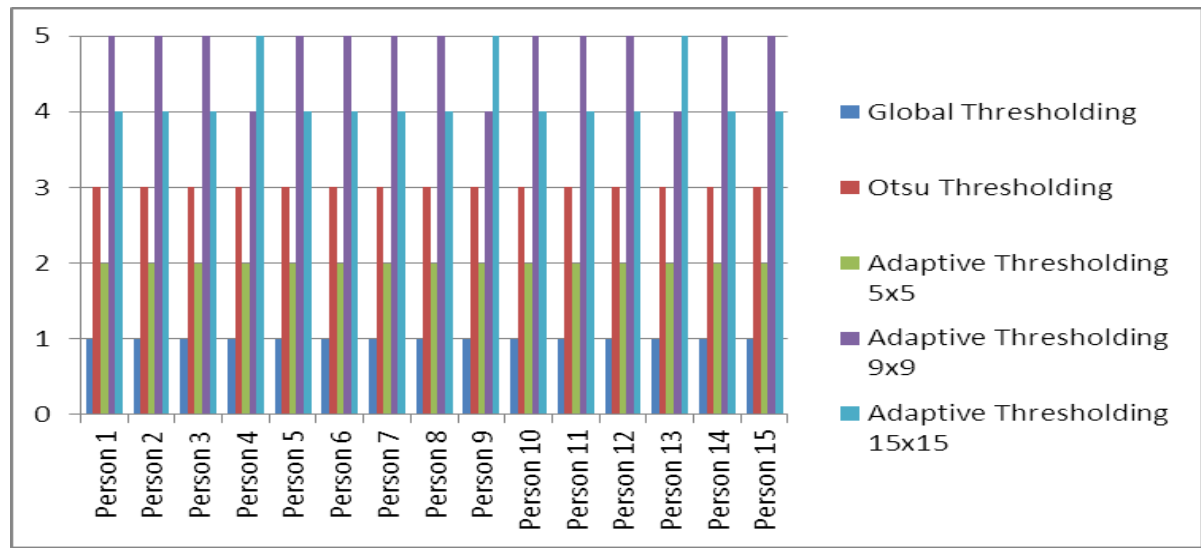

[Figure 1. Score Chart of Visual Analysis of an Image]

The result of the Criteria-I and Criteria-II are shown in Appendix-I.

\section{Criteria-III: Execution Time}

For mapping the performance of an algorithm the below Table 3 shows the average execution time (in milliseconds) for each binarization algorithm on all the images of each database. The Figure 2 shows the graphical representation of that data.

\begin{tabular}{|c|c|c|c|c|c|c|c|c|c|c|c|c|}
\hline \multirow[t]{3}{*}{ Algorithm } & \multicolumn{5}{|c|}{ FVC2000 } & \multicolumn{7}{|c|}{ FingerDOS } \\
\hline & & & & & & LEFT H & & & RIGHT & AND & & \\
\hline & DB1 & DB2 & DB3 & DB4 & $\begin{array}{l}\text { Average } \\
\text { Time }\end{array}$ & INDEX & MIDDLE & THUMB & INDEX & MIDDLE & THUMB & $\begin{array}{l}\text { Average } \\
\text { Time }\end{array}$ \\
\hline $\begin{array}{l}\text { Global } \\
\text { Thresholding }\end{array}$ & 210 & 175 & 170 & 140 & 174 & 290 & 150 & 140 & 135 & 150 & 170 & 173 \\
\hline $\begin{array}{l}\text { Otsu } \\
\text { Thresholding }\end{array}$ & 630 & 280 & 347 & 190 & 362 & 180 & 185 & 195 & 185 & 185 & 180 & 185 \\
\hline $\begin{array}{l}\text { Adaptive } \\
\text { Thresholding } \\
(\mathbf{9 x 9 )}\end{array}$ & 193 & 176 & 190 & 158 & 179 & 93 & 36 & 75 & 89 & 108 & 108 & 85 \\
\hline
\end{tabular}

[Table 3. Comparison of Execution Time for different algorithm] 


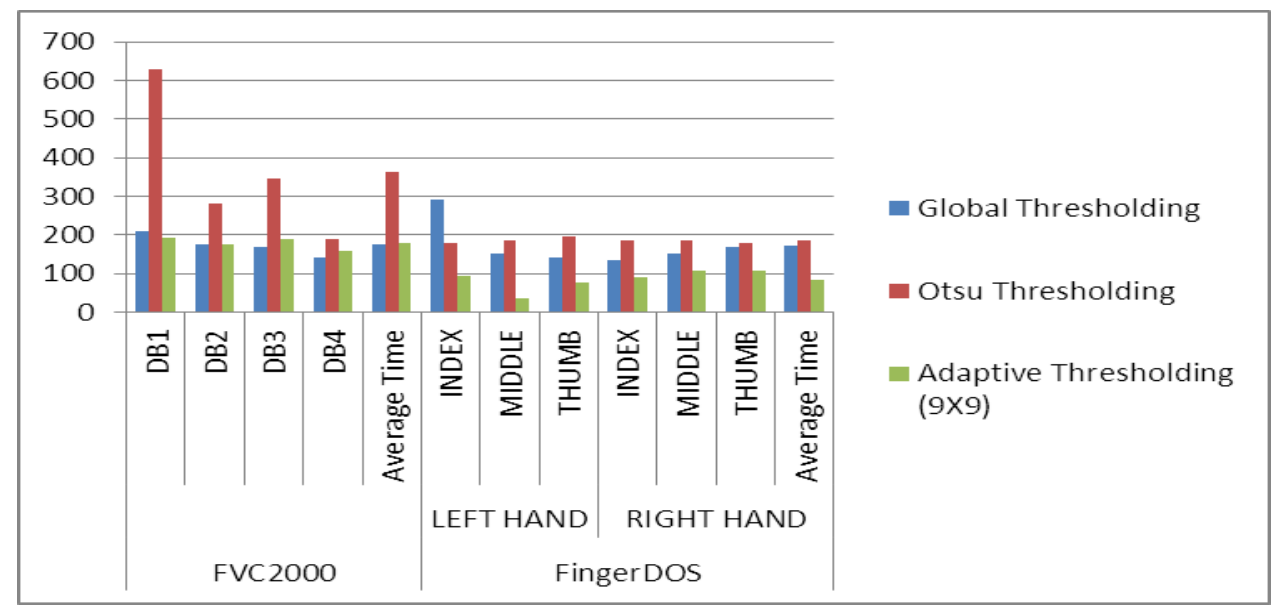

[Figure 2. Comparison of Execution Time for different algorithm]

After analyzing all the criteria, it does prove that the proposed adaptive thresholding improve the quality of an image as well as the performance.

\section{Conclusion And Future Work}

This paper describes the use of one of the most important pre-processing step binarization in fingerprint recognition. The feature extraction process is depending on the binarization and the thinning process. This paper shows the comparison of global and local thresholding technique. Also describe the proposed adaptive thresholding which use threshold value as a mean value of all the neighbor pixels. The proposed method is compare with others using different criteria like image quality, window size (e.g. 5x5, 9x9, 15x15), and execution time to measure the performance of the binarization process. The implementation is done using java on FVC2000 and FingerDOS database. The comparison show that proposed local adaptive thresholding using 9x9 matrix give the better result on all the criteria. After this we work on fingerprint thinning algorithm.

\section{References}

[1]. D Maltoni, D Maio, A K Jain, and A Prabhakar, "Handbook of Fingerprint Recognition,” Springer, New York, 2005

[2]. Woodard, J. D., Orlans, N. M., \& Higgins, P. T. (2003). Biometrics (electronic book). New York: McGraw-Hill/Osborne.

[3]. N. Otsu, "A threshold selection method from gray- level histogram," IEEE Transactions on System Man Cybernatics, Vol. SMC-9, No.1, pp. 62-66, 1979

[4]. Devi, H.K.A., (2006). Thresholding: A Pixel-Level Image Processing Methodology Preprocessing Technique for an OCR System for the Brahmi Script. Ancient Asia. 1, pp.161-165. DOI: http://doi.org/10.5334/aa.06113

[5]. A.S. Abutaleb, “Automatic Thresholding Of Gray-Level Pictures Using Two Dimensional Entropy”, Computer Vision, Graphics, And Image Processing, Vol.47, Pp.22-32, 1989.

[6]. J. Kittler and J. Illingworth, "Minimum Error Thresholding”, Pattern Recognition, Vol.19, No.1, Pp.41-47, 1986

[7]. K.H. Liang And J.J.W Mao, "Image Thresholding By Minimizing The Measures Of Fuzziness", Pattern Recognition, Vol.28, No.1, Pp.41-51, 1995

[8]. P. K. Sahoo, S. Soltani, and A. K. C. Wong, "A survey of thresholding techniques", Computer Vision Graphics Image Processing. $41,1988,233-260$

[9]. C. K. Chow and T. Kaneko, Boundary detection of radiographic images by a threshold method, in Proceedings, ZFZP Congress 71, pp. 130-134.

[10]. C. K. Chow and T. Kaneko, Automatic boundary detection of left ventricle from cineangiograms, Comput. Biomed. Res. 5, 1972, $338-410$.

[11]. S. M. X. Fernando and D. M. Monro, Variable thresholding applied to angiography, in Proceedings,6th International Conference on Pattern Recognition, 1982.

[12]. D. Maio, D. Maltoni, R. Capelli, J. L. Wayman And A. K. Jain, "Fvc2000: Fingerprint Verification Competition", Ieee Trans. Pattern Anal. Mach. Intell., Vol. 24, No. 3, Pp. 402-412, 2002.

[13]. F. Francis-Lothai And D. B. L. Bong, "Fingerdos: A Fingerprint Database Based On Optical Sensor," Wseas Transactions On Information Science And Applications, Vol.12, No. 29, Pp. 297-304, 2015.

[14]. K. Bhargavi, S. Jyothi ,"A Survey on Threshold Based Segmentation Technique in Image Processing", International Journal of Innovative Research \& Development, Vol-3, Issue-12,PP-234-239, 2014 
Appendix -I

\begin{tabular}{|c|c|c|c|c|c|}
\hline \multirow{2}{*}{ Original Image } & \multirow[t]{2}{*}{ Global Thresholding } & \multirow[t]{2}{*}{ Otsu Thresholding } & \multicolumn{3}{|l|}{ Adaptive Thresholding } \\
\hline & & & $5 * 5$ & $9 * 9$ & $15 * 15$ \\
\hline \multicolumn{6}{|l|}{ FVC2000 } \\
\hline 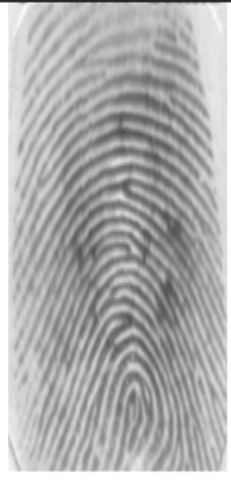 & (1) & ה) & (1) & (1) & (1) \\
\hline \multicolumn{6}{|l|}{ DBl (101_l.tif) } \\
\hline (1) & (1) & (a) & (3) & (1) & (1) \\
\hline \multicolumn{6}{|l|}{ DB2 (101_l,tif) } \\
\hline 年 & (1) & 年 & (2) & (1) & (1) \\
\hline \multicolumn{6}{|c|}{ DB3 (101_t.tif) } \\
\hline 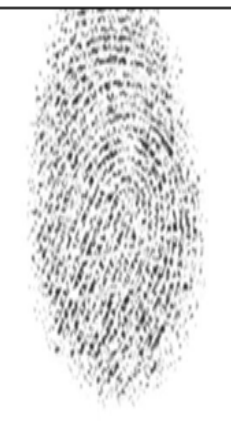 & 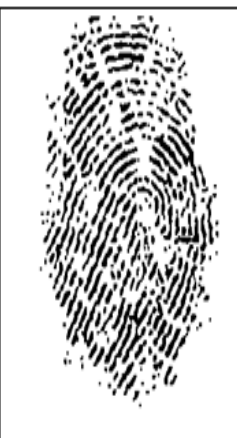 & 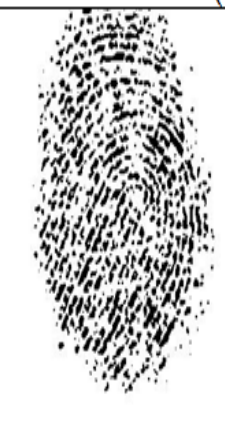 & 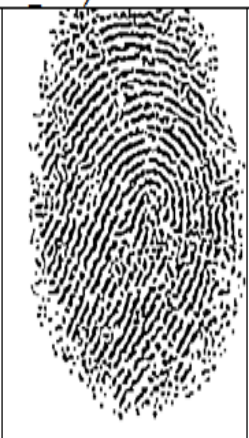 & 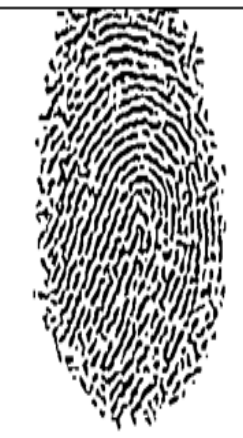 & 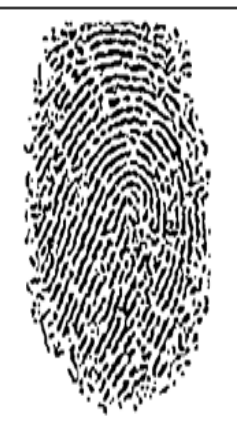 \\
\hline \multicolumn{6}{|c|}{ DB4 (101_1.tif) } \\
\hline
\end{tabular}


Performance Improvement in Binarization for Fingerprint Recognition

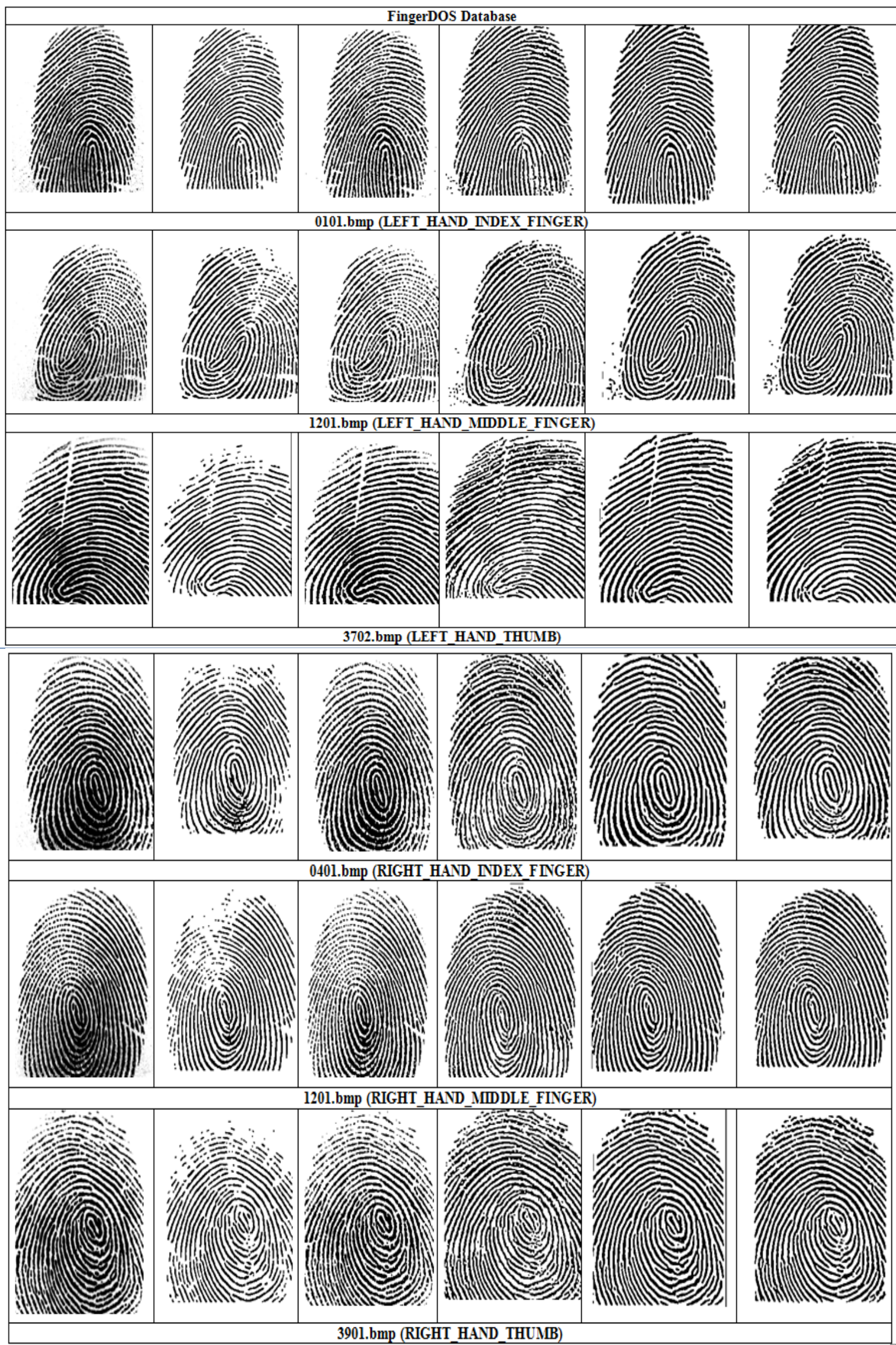

